\title{
Polarization of the transiting planetary system of the K dwarf HD 189733
}

\author{
Nadia M. Kostogryz ${ }^{1}$, Taras M. Yakobchuk ${ }^{1}$, Olexandr V. \\ Morozhenko $^{1}$ and Anatolij P. Vidmachenko ${ }^{1}$ \\ ${ }^{1}$ Main Astronomical Observatory NAS of Ukraine, \\ 27, Zabolotnoho str., Kyiv, 03680, Ukraine \\ email: kosn@mao.kiev.ua
}

\begin{abstract}
We model the polarization in the system HD 189733 resulting from the planetary transit. This system has a short-period $(2.2 \mathrm{~d})$ Jupiter-like planet with the radii ratio $R_{p} / R_{*}=$ 0.148 , orbiting at the distance of $0.031 \mathrm{AU}$ around the star.

We calculated the polarization of the system HD189733 to be $0.022 \%$ at the limb, which is consistent with the recent observational data. We suggest the shapes of the polarization parameters curves to be used for deriving the planet orbit inclination at the near limb transits as an alternative to standard transit method.
\end{abstract}

Keywords. polarization, methods: numerical, planetary systems

\section{Introduction}

During the last 1 - 2 decades a large number of extrasolar planets have been found and substantial effort is devoted to determination of the characteristics of these objects. However, for objects discovered by the most successful radial velocity method, a precise determination of the mass of the companion is not possible without the other techniques being applied (e.g. transits or astrometry). In this sense polarimetry comes as another promising technique (Hough et al. 2006; Keller 2006; Schmid et al. 2006) for studying the planetary atmospheres by means of scattered host star light, which provides information on their geometry, chemistry, and thermodynamics.

In this study, we model an effect that may be responsible for the polarization increase of the exoplanetary systems by $\sim 2 \times 10^{-4}$ based on Monte Carlo method proposed by Carciofi \& Magalhaes (2005).

\section{Results and Discussions}

HD 189733 is currently the brightest $\left(m_{V}=7.67 \mathrm{mag}\right)$ known star to harbor the transiting exoplanet (Bouchy 2005). This fact along with the short planetary period (2.2d) makes it very suitable for polarimetry. Since we are interested in measuring the occultation polarization effect, the ratio of planetary to star radii is also important parameter. The stellar radius of HD 189733 is equal to $0.788 R_{*}$ (Baines et al. 2009) and the planetary radius is $1.151 R_{J}$ (Southworth 2010), which resulting in ratio of 0.148 . The panel a) of Figure 1 illustrates the transit configuration of the system. Panel b) shows the time dependence of the stellar flux drop along the planet transiting. The next three panels c), d), e) demonstrate changes of Stokes parameters and polarization degree during occultation. Maximum polarization observed on the stellar limb is $\sim 2 \times 10^{-4}$. This value is very close to that obtained by Berdyugina et al. (2008) from direct observations, but authors ascribed this result to the light scattering in the planetary atmosphere. However, 
Lucas et al. (2009) concluded this effect cannot be explained by reflected light from the planet HD 189733b and explained it by the stellar spots only, given that HD 189733 is an active star. Based on our modelling, we argue that polarization of the system during planet transiting also cannot be rule out. Knowing precisely the moments of ingress and egress, this possibility can be verified by future polarimetric observations.

Until recently the only determination of inclination angle $i$ was based on the shape of the flux drop caused by the planet transit. One of the drawbacks of this method is that at smaller $i$ (i.e. high latitudes transits) flux signatures become weak and hard to detect. In contrast, the linear polarization during the occultation is more sensitive to the inclination angle. In particular, the maximum polarization does not depend on $i$, being defined by the ratio of planet-to-star radius only. This allows the planet orbit inclination to be determined even for the near limb transits.

\section{Conclusions}

In this paper we present the results of Monte Carlo simulations of the polarization in the planetary transiting system HD 189733 that ensues from the planet transit over the stellar disk. We derived two polarization maxima at the limb that amount to $\sim 0.022 \%$. The maximum polarization for HD189733 is very close to the value obtained by Berdyugina et al. (2008). Although they attributed this polarization to scattering of starlight by the planetary atmosphere, this is thought to be most unlikely (Lucas et al. 2009) and we suggest the polarization could arise from the planetary transit.

We find that the shapes of the polarization curves at the near limb transits can be used to determine the inclination of the planet orbit, more reliably than from the flux variation observations.

\section{References}

Baines, E. K., McAlister, H. A., Brummelaar, Th. A. T, Sturmann, J., Sturmann, L., Turner, N. H., \& Ridgway, S. T. 2009, ApJ, 701, 154

Berdyugina, S. V., Berdyugin, A. V., Fluri, D. M., \& Piirola, V. 2008, ApJ, 30, 490

Bouchy, F., Udry, S., Mayor, M., et al. 2005, A\& $A$, 444, L15

Carciofi, A. C. \& Magalhaes, A. M. 2005, ApJ, 635, 570

Hough, J. H., Lucas, P. W., Bailey, J. A., Tamura, M., Hirst, E., Harrison, D., \& Bartholomew-Biggs, M. 2006, PASP, 118, 1302

Keller, C. U. 2006, Proc. SPIE 6269,62600T-1

Lucas, P. W., Hough, J. H., Bailey, J. A., Tamura, M., Hirst, E., \& Harrison, D. 2009, MNRAS, 393, 229

Schmid, H. M., Beuzit, J.-L., Feldt, M., et al. 2006, Proc. IAU Coll.200, 165

Southworth, J. 2010, MNRAS, 408, 1689

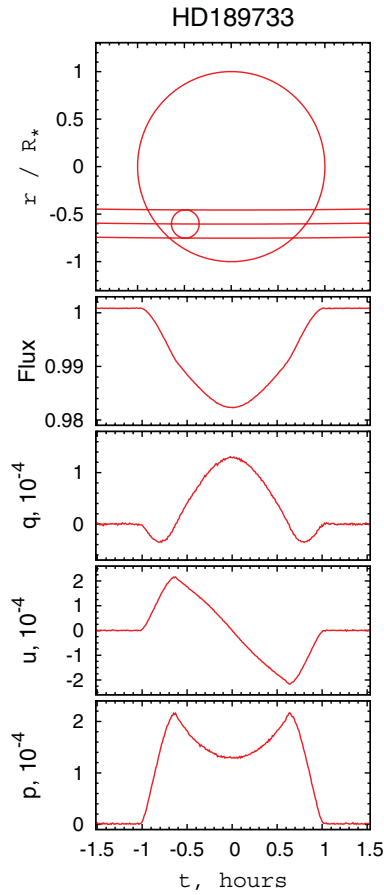

Figure 1. Modeling of the flux and polarization (Stokes $q=Q / I, u=U / I$ and polarization degree) for the planetary transit for HD189733. 